\title{
Biochemical and structural features of extracellular vesicle-binding RNA aptamers
}

\author{
KAZUYOSHI MURAKAMI, JING ZHAO, KAZUHIKO YAMASAKI and MAKOTO MIYAGISHI \\ Molecular Composite Medicine Research Group, Biomedical Research Institute, \\ National Institute of Advanced Industrial Science and Technology, Tsukuba-shi, Ibaraki 305-8566, Japan
}

Received January 12, 2017; Accepted February 16, 2017

DOI: $10.3892 /$ br.2017.899

\begin{abstract}
Extracellular vesicles are particles in mammalian body fluids that have attracted considerable attention as biomarkers for various diseases. In the present study, the authors isolated RNA aptamers with an affinity for extracellular vesicles from two library pools that encoded randomized sequences of different lengths. After the several rounds of selection, two conserved motifs are identified in the sequences that are obtained by next-generation sequencing. Most of the sequences were predicted to adopt a secondary structure that consisted of a non-conserved stem structure and a conserved loop sequence. Two minimal similar sequences are synthesized and confirmed the ability of these sequences to bind to extracellular vesicles. Circular dichroism spectroscopy and melting temperature analysis demonstrated that the aptamers were able to form a G-quadruplex structure in their loop regions and these structures were stabilized by potassium ions. Consistent with these structural data, the affinity of each aptamer for extracellular vesicles was dependent on potassium ions. The aptamers that were identified may be useful molecular tools for the development of diagnostic methods that utilize body fluids, such as blood, saliva and urine.
\end{abstract}

\section{Introduction}

Extracellular vesicles, which consist of lipid bilayer membranes, are secreted from various cells into mammalian body fluid, such as blood, saliva and urine (1). Such vesicles are generally classified into two groups: Exosomes and microvesicles, according to their size, physical properties and biogenesis (2). Exosomes are small particles with lipid bilayer membranes, ranging from 40 to $100 \mathrm{~nm}$ in diameter. They are associated with characteristic markers such as CD9,

Correspondence to: Dr Makoto Miyagishi, Molecular Composite Medicine Research Group, Biomedical Research Institute, National Institute of Advanced Industrial Science and Technology, 1-1-1 Higashi, Tsukuba-shi, Ibaraki 305-8566, Japan

E-mail: makoto.miyagishi@aist.go.jp

Key words: RNA aptamer, extracellular vesicle, next-generation sequencing, non-conserved stem structure, G-quadruplex sequence
CD63, CD81 and HSP70 and are derived from the endocytic recycling pathway. Microvesicles are extracellular particles of 100 to $1,000 \mathrm{~nm}$ in diameter that are produced directly from the plasma membrane as a consequence of outward budding. Thus, the markers associated with microvesicles are considered to depend predominantly on the receptors that are present on the surface of the cells from which they are derived.

Extracellular vesicles contain proteins, microRNAs, mRNAs and DNA from their parent cells $(3,4)$, and it appears that indicated these vesicles are important mediators of the transfer of information among cells (5). These features have suggested the potential utility of extracellular vesicles as diagnostic biomarkers of disease, as already proposed for ovarian cancer, lung cancer, pancreatic cancer and renal deficiency (6-10). Several methods have been developed for isolation of extracellular vesicles from biological samples, for example, ultracentrifugation, gel dialysis, immunoprecipitation, filtration and density-gradient centrifugation (11-15), but there is as yet no consensus as to optimal methods with respect to convenience and purity.

Aptamers are artificial DNA or RNA oligonucleotides that bind to targets with high affinity and specificity, and were initially investigated by Ellington and Szostak (16) and Tuerk and Gold (17) and their colleagues in the early 1990s. Aptamers can be selected using molecular-evolution technology, via in vitro selection or the systematic evolution of ligands by exponential enrichment (SELEX). Aptamers have been isolated that are specific for a large variety of targets, such as small molecules, nucleotides, peptides and proteins (18). In addition, live cells have been used as targets for selection of aptamers specific for receptors on cell surfaces (19-23).

Given that extracellular vesicles are more stable than cells and contain lipids, sugars and proteins within and/or on their membranes, as do cells themselves, the authors designed a procedure for isolation of RNA aptamers that bind to targets on extracellular vesicles, with the goal of developing new diagnostic systems. The current study reports the isolation of two aptamers specific for extracellular vesicles and the description of the target-specific affinity and structural features of these aptamers.

\section{Materials and methods}

Cell lines and preparation of extracellular vesicles. 293T cells were a generous gift from Professor A. Fukamizu (Tsukuba 
University, Tsukuba, Japan). HeLa S3 cells were obtained from the RIKEN BioResource Center (Tsukuba, Japan). Both cell lines were maintained in Dulbecco's Modified Eagle's medium (DMEM; Thermo Fisher Scientific, Inc., Waltham, MA, USA), supplemented with $10 \%$ fetal bovine serum (Invitrogen; Thermo Fisher Scientific, Inc.). For experiments, cells were transferred to DMEM without serum and cultured for 2 or 3 days. The resultant conditioned medium was collected and passed through a $0.22 \mu \mathrm{m}$ filter (EMD Millipore, Billerica, MA, USA) to remove cell debris. Then each sample was loaded into a $10 \mathrm{kDa}$ ultrafiltration cartridge (Amicon Ultra; EMD Millipore), subjected to these exchanges of buffer with $40 \mathrm{ml}$ PBS and concentrated to a final volume of $1 \mathrm{ml}$. The concentrated sample was placed in a 100 kDa ultrafiltration cartridge (VIVACON 500; Sartorius AG, Göttingen, Germany) and subjected to these exchanges of $500 \mu \mathrm{l}$ PBS. The protein concentration of each preparation of vesicles was determined in terms of absorbance at $260 \mathrm{~nm}$ using a NanoDrop spectrophotometer (Thermo Fisher Scientific, Inc., Wilmington DE, USA).

Selection of aptamers. Selection in vitro from two different library pools was performed as described previously (24-26). The libraries used are as follows: An N55 library that contained a region of 55 randomized nucleotides was used [5'-GGG AGG fUGG AAfC fUGA AGG AGA-(N55)-ACfU fUCG fCAA fUfCG fCfUfC fUAfC GfCA-3']; an N30 library that contained a region of 30 randomized nucleotides [5'-GGfU AGA fUAC GAfU GGA-(N30)-fCAfU G AfC GfCG fCAG fCfCA-3'], where f represents a 2'-fluoro modification. Selections were performed in binding buffer $[20 \mathrm{mM}$ Tris- $\mathrm{HCl}$ (pH 7.5), $150 \mathrm{mM} \mathrm{NaCl}, 5 \mathrm{mM} \mathrm{KCl,} 0.5 \mathrm{mM} \mathrm{MgCl}$ and $1.5 \mathrm{mM} \mathrm{CaCl}_{2}$ ].

Each pool of RNA was denatured at $98^{\circ} \mathrm{C}$ for $2 \mathrm{~min}$ and, following cooling, was incubated with extracellular vesicles in the presence of transfer (t)RNA $(100 \mu \mathrm{g} / \mathrm{ml})$ at room temperature. The mixture was passed through a $0.45 \mu \mathrm{m}$ HAWP nitrocellulose filter (EMD Millipore) and the filter was washed three times with binding buffer. The RNA that had bound to extracellular vesicles and was retained on the filter was recovered by incubation with $7 \mathrm{M}$ urea/10 $\mathrm{mM}$ EDTA at $98^{\circ} \mathrm{C}$ for $5 \mathrm{~min}$, extraction with phenol/chloroform and precipitation in ethanol.

Reverse transcription was performed with a PrimeScript II first-strand cDNA synthesis kit (Takara Biotechnology Co., Ltd., Dalian, China). Specifically, preparations of recovered RNA were supplemented with $1.25 \mu \mathrm{mol}$ dNTPs and 25 pmol each reverse primer [reverse primer N55, 5'-TGC GTA GAG CGA TTG CGA AGT-3'; reverse primer N30, 5'-TGG CTG CGC GTC ATG-3'] then incubated at $65^{\circ} \mathrm{C}$ for $5 \mathrm{~min}$. The RNase inhibitor (Takara Biotechnology Co., Ltd.) and $100 \mathrm{U}$ reverse transcriptase (Takara Biotechnology Co., Ltd.) were added to the solution, which was incubated at $30^{\circ} \mathrm{C}$ for $10 \mathrm{~min}, 37^{\circ} \mathrm{C}$ for $10 \mathrm{~min}, 42^{\circ} \mathrm{C}$ for $40 \mathrm{~min}, 52^{\circ} \mathrm{C}$ for $30 \mathrm{~min}$ and $98^{\circ} \mathrm{C}$ for $5 \mathrm{~min}$.

The cDNA was amplified by polymerase chain reaction (PCR) $\left(98^{\circ} \mathrm{C}\right.$ for $10 \mathrm{sec}, 55^{\circ} \mathrm{C}$ for $30 \mathrm{sec}$ and $72^{\circ} \mathrm{C}$ for $60 \mathrm{sec}$ ), with $10 \mathrm{mM}$ Tris- $\mathrm{HCl}$ (pH 8.3), $50 \mathrm{mM} \mathrm{KCl}, 1.5 \mathrm{mM}$ $\mathrm{MgCl}_{2}, 0.2 \mathrm{mM}$ dNTPs, $0.5 \mathrm{U}$ Ex Taq (Takara Biotechnology Co., Ltd.) and $0.125 \mu \mathrm{M}$ each forward primer (N55 forward primer, 5'-TGTA ATA CGA CTC ACT ATA GGG AGG TGG AAC TGA AGG AGA-3'; N30 forward primer, 5'-TGT AAT
ACG ACT CAC TAT AGG TAG ATA CGA TGG A-3') and reverse primer (N55 reverse primer, 5'-TGC GTA GAG CGA TTG CGA AGT-3'; N30 reverse primer, 5'-TGG CTG CGC GTC ATG-3'), and products were precipitated with ethanol. RNAs, whose pyrimidine residues were 2-fluoro modified, were transcribed from template DNAs with a DuraScribe ${ }^{\circledR}$ kit (Epicentre; Illumina, Inc., San Diego, CA, USA) at $37^{\circ} \mathrm{C}$ for $>6 \mathrm{~h}$ or overnight. Transcribed RNAs were treated with DNase I and purified on micro Bio-Spin columns (P-30; Bio-Rad Laboratories, Inc. Hercules, CA, USA) or by denaturing on $8 \%$ SDS-PAGE gels that contained $7 \mathrm{M}$ urea in Tris/borate/EDTA buffer (Wako Pure Chemical Industries, Ltd., Osaka, Japan). Then, they were subjected to the next round of selection.

Sequencing. Sequencing was performed with a MiSeq sequencing system (Illumina, Inc.) next-generation sequencer and the reagent kit v2 (Illumina, Inc.) exactly according to the manufacturer's protocol. The sequences obtained were ranked in order of decreasing frequency.

Predictions of secondary structure and search for consensus sequences. Secondary structures were predicted using the CentroidFold prediction program (National Institute of Advanced Industrial Science and Technology, Tokyo, Japan) for RNA secondary folding (27). Consensus sequence were identified using the motif-discovering algorithm and the MEME Suite 4.10.0 online server (http://meme.nbcr.net/ meme/tools/meme), applying the criterion of a minimum motif length of 8 nucleotides (28).

Minimized oligonucleotides. MO-1 [5'-GGC Cafc gafc gfcg gag gfug fugg ggg afufc gfuG GCC-3'] and MO-2 [5'-ggfu aga fuafc gafu gga aga ggg aaa ggg agg gfufu fcfua fcfc-3'] were purchased from Hokkaido System Science Co., Ltd. (Sapporo, Japan). Uppercase letters indicate deoxyribonucleic acids and lowercase letters indicate ribonucleic acids. The abbreviations fu and fc refer to 2-fluoro modified pyrimidines.

Filter-binding assay. For each assay, a 5'-biotinylated aptamer was incubated with extracellular vesicles in binding buffer that contained $100 \mu \mathrm{g} / \mathrm{ml} \mathrm{tRNA}$ at room temperature for $2 \mathrm{~h}$. The solution was passed through a nitrocellulose filter (EMD Millipore) and washed with $5 \mathrm{ml}$ binding buffer. The filter was exposed to UV irradiation $(254 \mathrm{~nm})$ and was blocked in binding buffer that contained 3\% BSA and $0.1 \%$ Tween-20 (Wako Pure Chemical Industries, Ltd.) for $60 \mathrm{~min}$. Following washing, the filter was incubated with streptavidin-horseradish peroxidase conjugate (Thermo Fisher Scientific, Inc.; cat. no. $\mathrm{N} 100$; dilution, 1:1,000) for $60 \mathrm{~min}$ at room temperature and washed. Then, the Amersham ECL Prime Western Blotting Detection reagent (GE Healthcare Life Sciences, Chalfont, UK) was used to visualize the chemiluminescence of the 5'-biotinylated aptamer on the filter.

Surface plasmon resonance (SPR) assay. SPR assays were performed with Biacore $X^{\mathrm{TM}}$ (GE Healthcare Life Sciences). Biotinylated oligonucleotides were immobilized on SA sensor chips (GE Healthcare Life Sciences). Then, extracellular vesicles were injected at the indicated concentrations (150, $75,50,43$ and $38 \mu \mathrm{g} / \mathrm{ml}$ ), and running buffer [binding buffer 


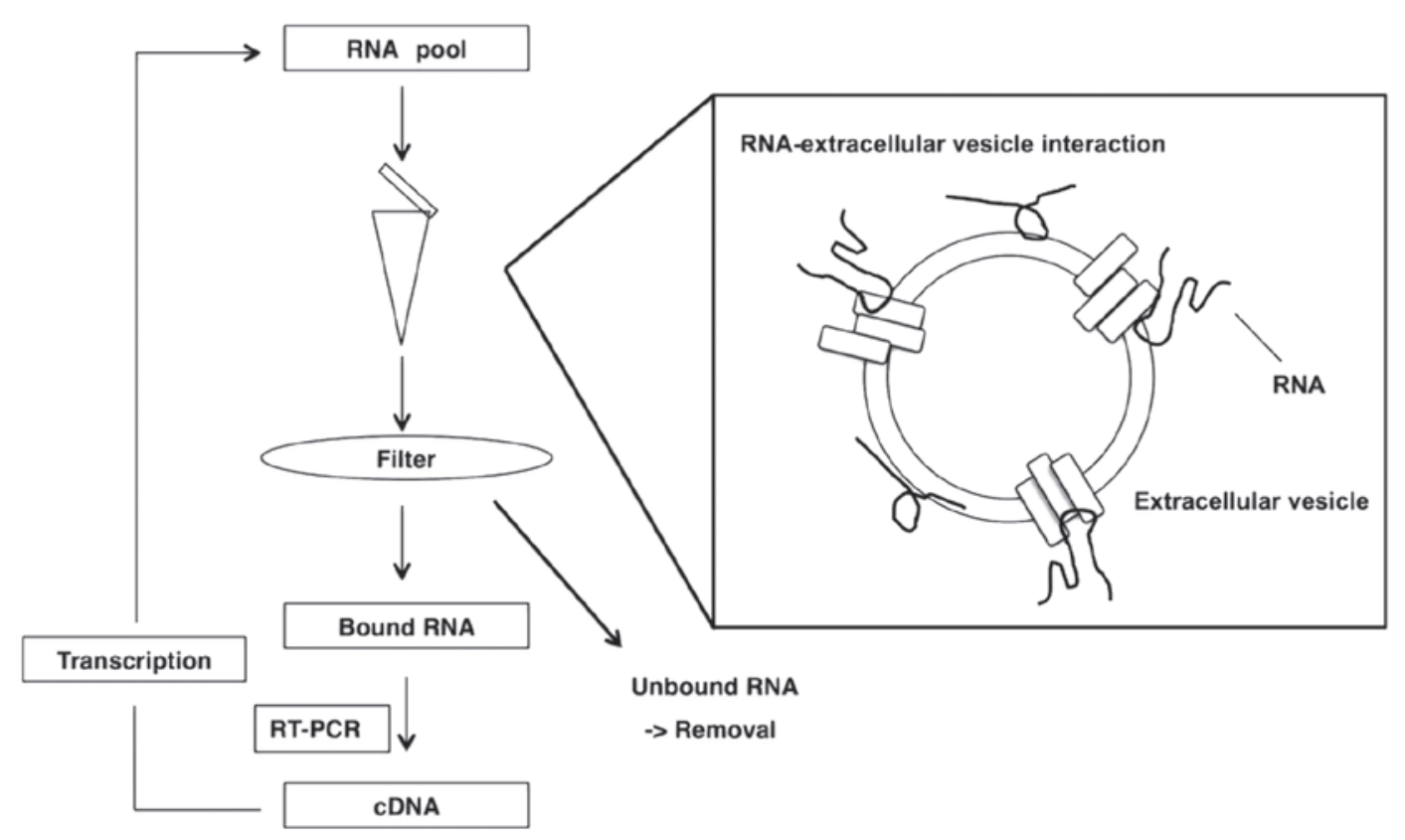

Figure 1. Schematic representation of enrichment by SELEX for aptamers that bind to extracellular vesicles. The two RNA libraries were incubated, separately, with extracellular vesicles. RNA-extracellular vesicle complexes were added to nitrocellulose filters, which were then washed repeatedly. The bound RNAs were eluted, purified and subjected to reverse transcription and amplification (RT-PCR). Pools of enriched RNA were generated by transcription from the products of PCR and used for the next round of selection. SELEX, systematic evolution of ligands by exponential enrichment; RT-PCR, reverse transcription-polymerase chain reaction.

supplemented with $0.005 \%$ Tween-20 (Wako Pure Chemical Industries, Ltd)] was loaded onto flow cells 1 (blank) and 2 (aptamer) of the sensor chip. Data were analyzed with Biacore $^{\mathrm{TM}}$ evaluation software (GE Healthcare Life Sciences; version, 2.0). following subtracting the background (flow cell 1) from values for flow cell 2 .

Prediction of G-quadruplex structure and measurements of circular dichroism (CD) and melting temperature (Tm). The presence of a G-quadruplex structure in individual aptamers was predicted by the QGRS Mapper (http://bioinformatics. ramapo.edu/QGRS/index.php) (29).

Circular dichroism (CD) spectra were recorded with a spectropolarimeter (J-820; Jasco International Co., Ltd., Tokyo, Japan) as described previously (30). A cell with a $1 \mathrm{~mm}$ path length was used for analyses of titrations with $\mathrm{KCl}$ (at $0 \mathrm{mM}, 0.1$, $0.3,1,5,10,50$ and $100 \mathrm{mM}$ ) in $20 \mathrm{mM}$ Tris-HCl buffer ( $\mathrm{pH} 7.5$ ). Spectra were recorded four times, from 220 to $320 \mathrm{~nm}$ at $25^{\circ} \mathrm{C}$. Then, $C D$ intensities were expressed in terms of $[\theta]$ per residue.

Melting temperatures of aptamers were determined at $270 \mathrm{~nm}$ in buffer that included $100 \mathrm{mM} \mathrm{KCl}$ as the temperature was raised from 25 to $95^{\circ} \mathrm{C}$.

Fractionation of extracellular vesicles and dot-blotting assay. Preparations of extracellular vesicles were fractionated on a column of sepharose 2B (GE Healthcare Life Sciences). Protein concentrations were determined with a DC protein assay kit (Bio-Rad Laboratories, Inc.) according to the manufacturer's instructions. Then, $2 \mu \mathrm{l}$ aliquots of fractionated and concentrated extracellular vesicles were spotted on a nitrocellulose membrane, which was dried for $30 \mathrm{~min}$ at room temperature.

For detection of binding of aptamers, the membrane was blocked with binding buffer that contained $1 \%$ BSA and $100 \mu \mathrm{g} / \mathrm{ml}$ tRNA for $60 \mathrm{~min}$ at room temperature. The membrane was then incubated with $100 \mu \mathrm{M}$ 5'-biotinylated aptamer in binding buffer supplemented with $0.1 \%$ Tween-20, $1 \% \mathrm{BSA}$ and $100 \mu \mathrm{g} / \mathrm{ml}$ tRNA for $60 \mathrm{~min}$ at room temperature and washed three times with binding buffer supplemented with $0.1 \%$ Tween-20. The membrane was exposed to UV irradiation $(254 \mathrm{~nm})$ and then to streptavidin-horseradish peroxidase conjugate (Thermo Fisher Scientific, Inc.; cat. no. N100; dilution, 1:4,000) for $60 \mathrm{~min}$ at room temperature. It was washed three times and, finally, the Amersham ECL Prime Western Blotting Detection reagent (GE Healthcare Life Sciences; cat. no. RPN2232) was used to visualize the chemiluminescence of the aptamer on the membrane.

For detection of antibodies, the membrane was blocked in PBS supplemented with $0.1 \%$ Tween-20 (PBS-T) and containing $3 \% \mathrm{BSA}$ for $60 \mathrm{~min}$ at room temperature. The membrane was incubated with human CD63-specific mouse monoclonal antibody (Santa Cruz Biotechnology, Inc., Dallas, TX, USA; cat. no. Mx-49.129.5; dilution, 1:1,000) for $60 \mathrm{~min}$ at room temperature and washed three times with PBS-T. The membrane was exposed to second antibody-HRP conjugate (GE Healthcare Life Sciences; cat. no. NA931; dilution, 1:2,000) for $60 \mathrm{~min}$ at room temperature and washed three times. Finally, the Amersham ECL Prime Western Blotting Detection reagent (GE Healthcare Life Sciences; RPN2232) was used to visualize chemiluminescence.

\section{Results}

Isolation of RNA aptamers with affinity for extracellular vesicles. To isolate RNA aptamers with an affinity for extracellular vesicles, the authors used a selection method in vitro that was based on a previously reported method (Fig. 1) (24-26). 
A

Consensus sequence-1 in S pool

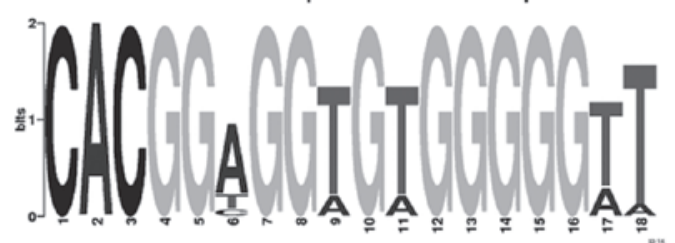

Consensus sequence- 1 in $\mathrm{K}$ pool

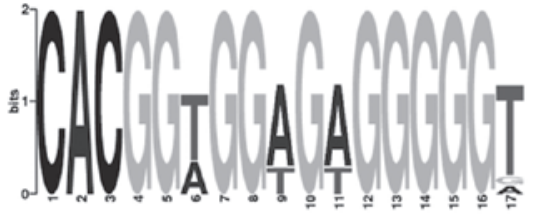

Consensus sequence- 2 in $\mathrm{K}$ pool

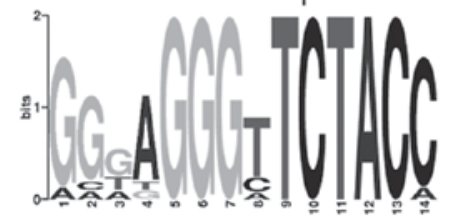

B
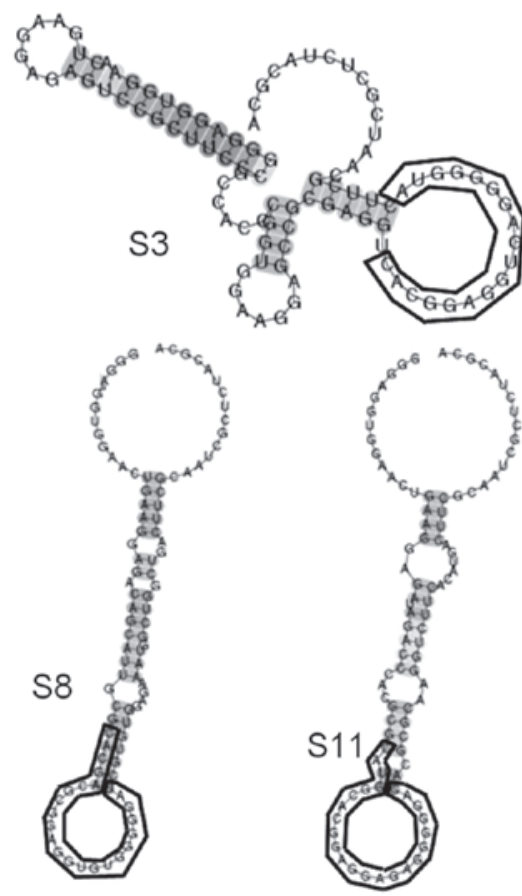

C
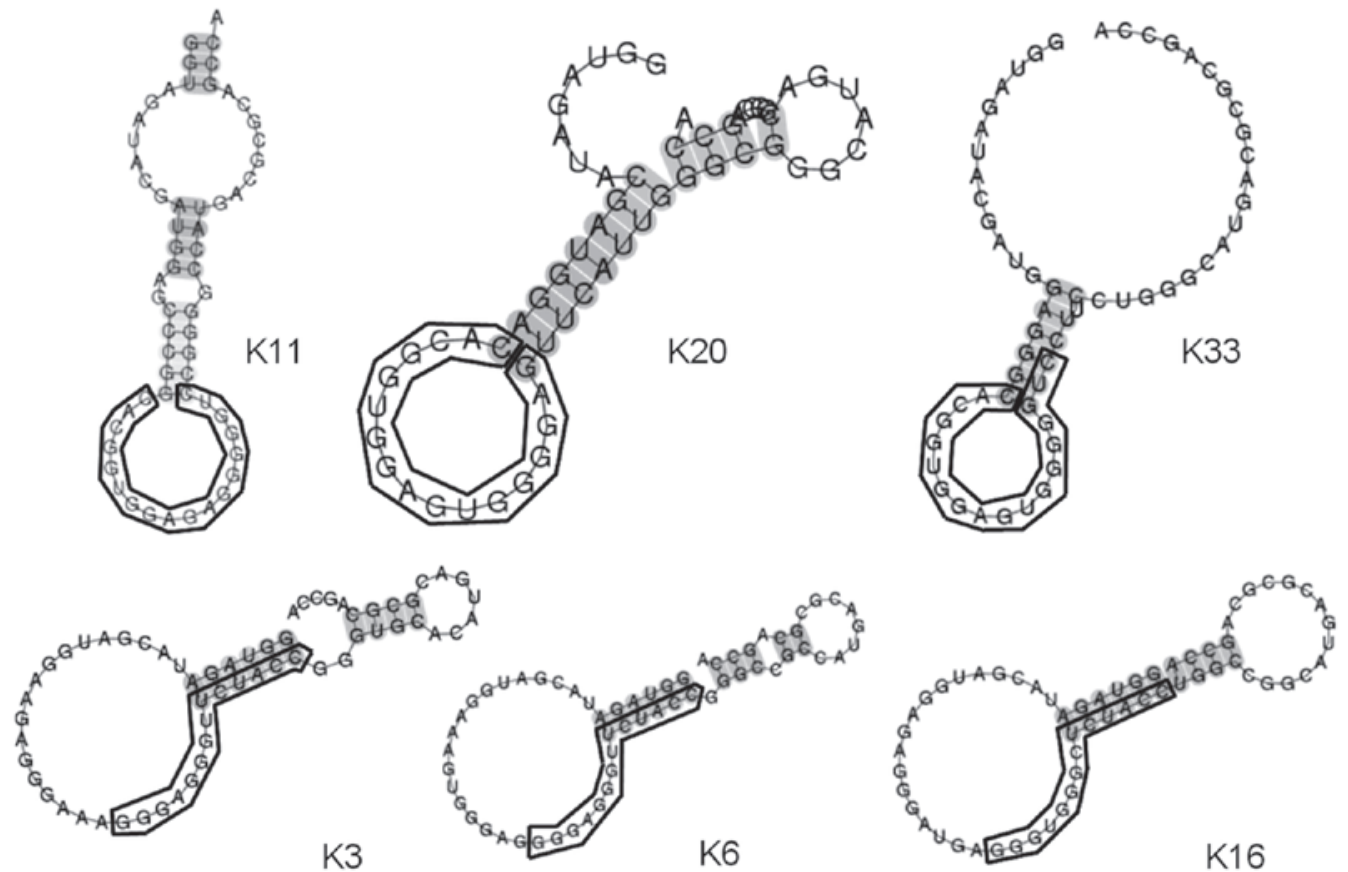

D
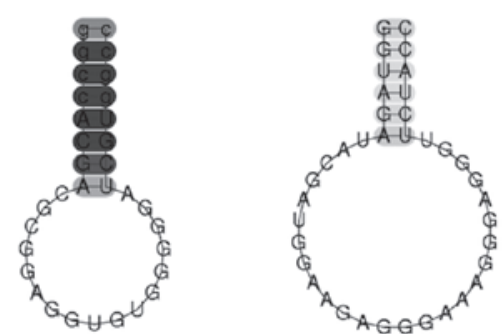

E

Extracellular vesicles
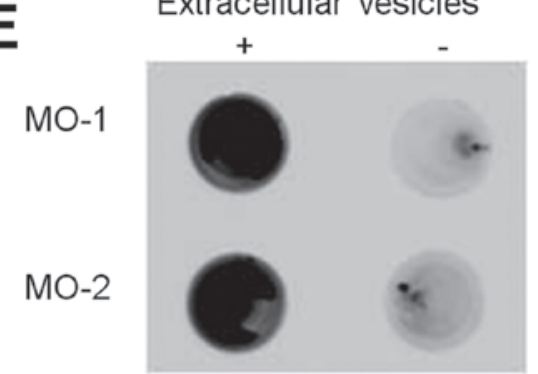

Figure 2. Characterization of aptamers that bound to extracellular vesicles. (A) The consensus sequences shared among the top 50 selected sequences, as identified with the MEME suite. (B and C) Typical secondary structures (clones S3, S8 and S11 in the S pool and clones K3, K20, K33, K3, K6 and K16 in the $\mathrm{K}$ pool), as predicted by the CentroidFold program. Consensus sequences are enclosed in boxes. (D) Secondary structures of minimized aptamers MO-1 and MO-2. (E) Binding of selected aptamers to extracellular vesicles. Labeled aptamers (MO-1 and MO2) were incubated with extracellular vesicles and mixtures were filtered through a membrane. Aptamer-extracellular vesicle complexes were trapped on the membrane (+). The minus sign (-) represents negative controls without aptamers. MO, minimized oligonucleotides. 
A 97-nt RNA library pool (N55 pool) and a 60-nt RNA library pool (K pool) was prepared, with respective central randomized 55 and 30-nt sequences, by in vitro transcription with 2'-fluoro-modified pyrimidines from synthesized DNA libraries, as templates. Selection was performed as follows. A transcribed RNA library pool was incubated with extracellular vesicles in binding buffer for $30 \mathrm{~min}$. The RNA-extracellular vesicle complexes were added to a nitrocellulose filter and washed. Bound RNAs were eluted, purified and subjected to reverse-transcription and amplification. An enriched RNA pool was transcribed from the amplified products of PCR and used for the next round of selection.

After 10 rounds of selection, the enriched pools were sequenced from the eighth, ninth and tenth rounds using a next-generation sequencer. The 50 most abundant unique sequences were ranked according to frequency and the results are presented in Tables I and II.

The MEME suite (28) was used to search for sequence similarities among all 50 sequences from the respective tenth rounds of enrichment (Fig. 2A). The analysis revealed two common sequences, the consensus sequence-1 'cac gg(t/a) $\operatorname{gg}(\mathrm{t} / \mathrm{a}) \mathrm{g}(\mathrm{t} / \mathrm{a}) \mathrm{g} g \mathrm{gg} \mathrm{gt}^{\prime}$ in the $\mathrm{S}$ pool (31 out of 50 clones), the $\mathrm{K}$ pool (5 out of 50 clones) and the consensus sequence- 2 'ggg agg gtt cta $\mathrm{cc}$ ' in the $\mathrm{K}$ pool (17 out of 50 clones), respectively. Consensus sequence-1 was identified from both library pools, even through the design of the two libraries (lengths of randomized region and the fixed primer regions) were different. The independent isolation of consensus sequence- 1 from the two very different libraries suggested that the sequence may have strong affinity for extracellular vesicles.

Next, the secondary RNA structures of the complete sequences of 53 clones were analyzed ( 36 clones that included consensus sequence-1 and 17 clones that included consensus sequence-2) with primer regions, using CentroidFold software, to identify common secondary structures (Fig. 2B) (27). All of the sequences were predicted to have a non-conserved stem with a loop. Some examples of this structure are presented in Fig. 2C.

Next, the authors attempted to shorten a full-length sequence to a minimized sequence with the step-loop structure (Fig. 2D). The sequences of clone S8 and clone K3 were selected as representatives of aptamers with each respective consensus sequence because both were in the upper ranks of respective rankings and were likely to be relatively stable as a result of their longer stems. The authors synthesized minimized oligonucleotide-1 (MO-1), including consensus sequence-1 referenced to clone $\mathrm{S} 8$, and minimized oligonucleotide-2 (MO-2), including consensus sequence-2 referenced to clone $\mathrm{K} 3$, respectively.

To examine the binding affinities of MO-1 and MO-2 for extracellular vesicles, filter-binding assays were performed with the respective 5'-biotinylated minimized aptamers. Both aptamers bound to the extracellular vesicles (Fig. 2E) and not to BSA or mouse IgG (data not shown), indicating that the minimized sequences had retained binding affinity and specificity with respect to extracellular vesicles.

Analysis of interactions of aptamers with extracellular vesicles. To examine the interaction of the each minimized aptamer with extracellular vesicles, affinities were measured by SPR. The 5'-biotinylated minimized oligonucleotides were immobilized on streptavidin-coated sensor chips. Extracellular vesicles at various concentrations were then passed over the immobilized oligonucleotides. SPR analysis revealed that MO-1 bound to extracellular vesicles with a $\mathrm{K}_{\mathrm{D}}$ of $1.17 \times 10^{-15} \mathrm{mg} / \mathrm{ml}$, while $\mathrm{MO}-2$ bound to extracellular vesicles with a $K_{D}$ of $6.08 \times 10^{-16} \mathrm{mg} / \mathrm{ml}$ (Fig. 3). These rather similar values suggested that two aptamers may bind to the same target on extracellular vesicles.

Since both aptamers bound to extracellular vesicles derived from $293 \mathrm{~T}$ cells, the authors examined whether the aptamers could bind to extracellular vesicles derived from another cell line. Extracellular vesicles were prepared from HeLa S3 cells and the SPR analysis was repeated. The results demonstrated that the MO-1 aptamer bound to HeLa S3 extracellular vesicles with a $\mathrm{K}_{\mathrm{D}}$ of $6.83 \times 10^{-15} \mathrm{mg} / \mathrm{ml}$, and the MO-2 aptamer bound to the HeLa S3 extracellular vesicles with a $\mathrm{K}_{\mathrm{D}}$ of $2.82 \times 10^{-16} \mathrm{mg} / \mathrm{ml}$. These results were similar to those obtained with 293T-derived extracellular vesicles. Thus, these observations suggested that the two aptamers may recognize a common target on extracellular vesicles from a variety of sources.

Spectroscopic analysis of the structures of minimized aptamers. There are many examples of guanine-rich sequences folding into G-quadruplex structures in the presence of potassium ions $(31,32)$. In previous studies of the authors, it was demonstrated that an RNA aptamer directed against a bovine prion forms a G-quadruplex structure (25,26,33-35). Since guanine-rich sequences were present in the MO-1 and MO-2 aptamers, the authors examined whether the guanine-rich sequences may resemble the G-quadruplex consensus motif, using the QGRS Mapper, namely, G-quadruplex analysis software (29). There was a high probability of the presence of a G-quadruplex structure in both the MO-1 aptamer $(\mathrm{G}$-score $=19)$ and the MO-2 aptamer $(\mathrm{G}$-score=20).

To confirm these predictions experimentally, structural and thermodynamic analyses were performed of each aptamer by circular dichroism (CD) spectroscopy and thermal melting point (Tm) analysis (Fig. 4A). Both aptamers yielded a negative band at $240 \mathrm{~nm}$ and a positive band at $260 \mathrm{~nm}$ in the CD spectrum. The minimum band at $240 \mathrm{~nm}$ and the maximum band at $260 \mathrm{~nm}$ were induced by potassium ions in a concentration-dependent manner ( $\mathrm{K}^{+}$from $0 \mathrm{mM}$ to $100 \mathrm{mM}$; Fig. 4A). The $\mathrm{T}_{\mathrm{m}}$ values of the aptamers were relatively high (approximately $70^{\circ} \mathrm{C}$; Fig. 4B), indicating that the aptamers were tightly folded, as expected from a G-quadruplex structure. All of the results supported the hypothesis that both aptamers were folded into a G-quadruplex.

G-quadruplex structures have been classified into two characteristic spectral forms (36) on the basis of their CD spectra: The parallel G-quadruplex with a maximum at $260 \mathrm{~nm}$ and a minimum at $\sim 240 \mathrm{~nm}$, and the anti-parallel G-quadruplex with a maximum at $\sim 290 \mathrm{~nm}$ and a minimum at $\sim 260 \mathrm{~nm}$. Both the MO-1 and the MO-2 aptamers yielded a maximum at $\sim 260 \mathrm{~nm}$ and a minimum at $240 \mathrm{~nm}$, strongly suggesting the presence of a parallel G-quadruplex in each one (25,26,33-35), presented schematically in Fig. 4C.

Next, the binding of aptamers to extracellular vesicles in the presence and absence of potassium ions was examined. SPR 
Table I. The sequences obtained from S pool.

\begin{tabular}{|c|c|c|}
\hline Clone & Number & Sequence $\left(5^{\prime}-3^{\prime}\right)$ \\
\hline S1 & 935 & ACGCTAGCTGTGGAAAGACGCTAAATCGGGAGGTGGGTTGGGGTGCTAGCGATCA \\
\hline S2 & 375 & CTAGGGTGGGGAAATATGGAGGGCGCTCAGGGCTACACTGACTACGTGATTAGAG \\
\hline S3 & 365 & GTCCGCTTCCCGCCACCCGGTGGAAGGAGCCGCGAGGTCACGGAGGTGAGGGGGT \\
\hline S4 & 362 & TGCTCATTCGTCATAGGTGGAGGGTAGTAGGGGGCGAACAGTTCCACGCTAGCGT \\
\hline S5 & 357 & GGTGGGTTATAGAGGTTGGGTCCCCGACGTTGCTTCCCTATATAGAGTGGCGAGG \\
\hline S6 & 344 & TCTCTCGCAGCGGTCTGCATGGTAGCGGGTCAACCACGGAGGAGTGGGGGTGTTG \\
\hline S7 & 310 & CACGGAGGTGTGGGGGTTCTCAAAACGTTCTATGCTTGGCCAATCCCGAGCCGTC \\
\hline S8 & 223 & CAGCATTGCGCACGACGCGGAGGTGTGGGGGATCGTGTGGAGAAATGGCTGGCTG \\
\hline S9 & 180 & ATGGGATGGGAATAGTTCACCACAATTTTTGGGCTCCTTAGCTACACGCGATGGA \\
\hline $\mathrm{S} 10$ & 169 & AGCAACGAAAATTACTGCCGCGGAGGAGAGGGGGAGCAGTATTCGTTGGGTCATA \\
\hline S11 & 137 & TAGACCCACGCGCATGGCACGGAGGAGAGGGGGACACGCGCAAGGTCTTCACATG \\
\hline S12 & 134 & AAATTCGCGTTGTAGGTGCACGGTGGTGTGGGGGTTATCCGGCAACGCGTTTGGT \\
\hline S13 & 124 & GGTGCACCATCTCCGGTCTCGCGCGGTGGAGTGGGGGTTGAGACCGGGGTTGGTT \\
\hline S14 & 107 & CTTACTCGCAGACACGGAGGTGTGGGGGTTCTGTGGGAGCTTGCACGAGACCTTC \\
\hline S15 & 98 & TGAGTTAGTGGTGCTGCGCGCGGAGGAGTGGGGGTTGCAGCGTTACGTACGAGTC \\
\hline S16 & 80 & TGCCGTGTCCGGAATTGCACGGAGGTGAGGGGGATATATTCTGGATAGCGCGCGG \\
\hline S17 & 78 & TTTGGGGCGTTTCATCAAGATTCGGGTTGGGGACTCTAATAGTAGCCGGTTGTGG \\
\hline S18 & 78 & ATTTTCGAATCCGCGTCCCGGCGGATAGCACGCGAGGTTACGGCGGAGTGGGGAA \\
\hline S19 & 77 & CACGGAGGTGTGGGGGTTCTCAAAACGTTCTATGCTTGGCCAATCCCGAGCGGTC \\
\hline S20 & 77 & ACTGTTGAACGCTCACGTGTACGGAGGTGTGGGGAATACGTGGGGGGTAACATCT \\
\hline S21 & 75 & CACGGCGGAGTGGGGAGACTCTTGAAATGTTCCGGGTTGCGAACGTCGGTGCTGG \\
\hline S22 & 71 & CACCGGGGGCAACATGGCGCACGGAGGAGAGGGGGTCGTCTAGTTAGTCCCGGTG \\
\hline S23 & 63 & TGGCGCGGGTTCGCTGACACGGCGGTGTGGGGGTTCAGGAAACAATGCGCACGAA \\
\hline S24 & 63 & GGTATTGGGCGGGGGTTCCCAAAAGTAGCGCGACGTCATACGAAGCCGTACGTCG \\
\hline S25 & 62 & GGCGGAGCACGTATTGACACGGTGGAGAGGGGGTTCATACGGCTCAGGCACCTAA \\
\hline S26 & 56 & TCGGGACGGCCTGAGGCGCACGGTGGTGTGGGGGTTGCCTACTGCCGTTTGGTCA \\
\hline S27 & 54 & TCTCTCGCAGCGTCTGCATGGTAGCGGGTCAACCACGGAGGAGTGGGGGTGTTG \\
\hline S28 & 43 & GAGCGCAATCTAACACGGAGGTGTGGGGGTTTAGCGTGCGCAAACTCAGATGCGT \\
\hline S29 & 38 & CTCAGTTCATCGTCCCTAGACGCACGGCGGAATTGGGAGTACGGAGGTGGGGGGA \\
\hline S30 & 38 & ACGAAGTTTAGGATCGCTCCTTCCACGGCGGTGAGGGGGTGAAGCTGCGACCCAG \\
\hline S31 & 38 & AAGCCGCATCCGGCGGGCGACACGGAGGAGAGGGGGATCGCCACAGCCTGCGGTG \\
\hline S32 & 36 & TTGGAGGGGGTGTGCCTATTTGGGGTTCAGCAGGGGCACATACGCGGTTGACGAG \\
\hline S33 & 35 & CGTCAATCACGGCGGTGAGGGGGCATTGATGTCACGCAAAGTAGGCCTAATACCC \\
\hline S34 & 29 & CACGGAGGAGTGGGGGTTCTCTGGGAAGTTTCGTCTTGTTGGTACGTAACCCGGC \\
\hline S35 & 28 & AGGCCCGTACGGAGGTGTGGGGATTGGGTCTTTTGACTTGCGAGGCCGTTGTGCG \\
\hline S36 & 27 & ACGCTAGCTGTGGAAAGACGCTAAATCGGGAGGTGGGTTGGGGTGCTAGCGATCG \\
\hline S37 & 26 & TGAGGGATGGCCACGGAGGAGTGGGGGTGCCATCGCAACGTTGACACGGGTTGCA \\
\hline S38 & 26 & GTGTGCCGAGCGATAGGGACACGGTGGAGTGGGGGGACCTATGCCCGGCAAGACA \\
\hline S39 & 26 & GGGGTCATTAGGAGGGGCCTTTAGAAAAATAGTAGCCGCTGCGGGTCCTTTCGGG \\
\hline S40 & 24 & ACGCTAGCTGTGGAAAGACGCTAAATCGGGAGGTGGGTTGGGGTGCTAGTGATCA \\
\hline S41 & 21 & GATTTGGGGGCCACGGAGGTGAGGGGGCGCTCCTATTCTCGTGTTTTGCGTGCGA \\
\hline S42 & 21 & CGGGCGCCGGGTTAGATCACGGAGGTGTGGGGGTATCTACCCCGTGAGGCGCCAC \\
\hline S43 & 20 & GTGTTGCACCCGCCTGGGGCACGGAGGTGTGGGGGATCCCAGGGTGAGCGACTAA \\
\hline S44 & 20 & CTAGGGTGGGGAAATATGGAGGGCGCTCAGGGCTACACTGACTATGTGATTAGAG \\
\hline S45 & 20 & ATTTTTTTAATGCATCATTTTTACACTCCTTTTGGACCAACCCAACGGGCGCTGC \\
\hline S46 & 19 & CACTCGGGTTGGAGGCGCACGGCGGTGTGGGGGTCGCTTCAAGTGACGGGTGTCA \\
\hline S47 & 18 & TGCACAGGTGACACGCCGATCACGGAGGTGTGGGGAGATCGGCAATGGCACGGTG \\
\hline S48 & 18 & GTCGAAGTGTAACTTATTTGTGTGATTTTTTTGTTTTATGCTTACACGCGGCTCA \\
\hline S49 & 18 & GGTGGGTTATAGAGGTTGGGTCCCCGACGTTGCTTCCCTATATAGAGTGGCGCGG \\
\hline S50 & 18 & ATTTTGTTTTGTTGTTTTTGTGTACTCCTTGAGATGCTGGCTACTGTCCGAGCCG \\
\hline
\end{tabular}


Table II. The sequences obtained from K pool.

\begin{tabular}{|c|c|c|}
\hline Clone & Number & Sequence $\left(5^{\prime}-3^{\prime}\right)$ \\
\hline K1 & 918 & ATAGCGGGAGGGAGGGTTCTACCTGGTGGG \\
\hline $\mathrm{K} 2$ & 692 & GGGTGGAGGGAAGGAGTGGGGTTCTACCGG \\
\hline K3 & 645 & AGAGGGAAAGGGAGGGTTCTACCGGGTGCA \\
\hline K4 & 552 & ACGTGGGAGGGATTGGGGTATCTCCGGTTG \\
\hline K5 & 475 & AGAGGGTGGGAAAGGGTTCTACCACAGTGC \\
\hline K6 & 471 & AAGTGGGAGGGGAGGGTTCTACCGGGCCGC \\
\hline K7 & 436 & GTAGGGAGGTATGTATCTCCTGGTTGGGGG \\
\hline K8 & 403 & GAGGGAAGGGATATGGGGTATCTAGGGCCG \\
\hline K9 & 367 & AAGAGGGAGGGTAGGGTTCTACCAGCTGGG \\
\hline K10 & 361 & GTACGGAGGTGAGGGGAACTCCACGGTCGG \\
\hline K11 & 356 & GCCCGGCACGGTGGAGAGGGGGTCCGGGGC \\
\hline K12 & 355 & AGTGGGACTGGGATGGGGTGTATCGCCCGG \\
\hline K13 & 349 & ATGAGGGAGGGTTGGGGTATCTCCCCGGTG \\
\hline K14 & 338 & TGAGGGAATAGGGAAAGGGGTATCGTTGGG \\
\hline K15 & 287 & ACGAGGGAGGGAGGGGTATCACCGGGCCGG \\
\hline K16 & 276 & GAGGGATGAGGGTGGGCTCTACCTGGCCGG \\
\hline K17 & 271 & GCCTACGCACGGTGGAGAGGGGGTTGTGGG \\
\hline K18 & 236 & AGTGGGAAGGGTTTGGGTGGTCTACCGTGG \\
\hline K19 & 230 & GAGGGAGGGAGGGCTCTACCTTTGTGGCCC \\
\hline K20 & 225 & CACGGTGGAGTGGGGAGTTCATTGGGCGGG \\
\hline $\mathrm{K} 21$ & 222 & AGTGGGATGGGTAGGGTTCTACATATGCTG \\
\hline K22 & 207 & GCGGGATTGGGTTTTGGGGTATCTGGGCGG \\
\hline K23 & 205 & CGGTCAATGCCCACGGTGGAGAGGGGGTGG \\
\hline K24 & 188 & CCACGGAGGTGAGGGGGTGTCCACGGTGGC \\
\hline K25 & 185 & TAGAGGGAGGGAGGGATCTACCAGGTGGGG \\
\hline K26 & 174 & TAGTGGGAGGGAATGGGATTTCTACCGGGG \\
\hline K27 & 170 & CACTGGAGGAGGGTGGGGAGTTCATCCGGG \\
\hline K28 & 158 & TTGAGGGAAAGGGTGGGGCATCTACCGTGG \\
\hline K29 & 151 & CCGTAACACGGAGGAGAGGGGGGAACGGTG \\
\hline K30 & 146 & CGCGGAGGTGTGGGGGATCCGTCGTGGTGG \\
\hline K31 & 149 & GAGGGAGGGACTGGGGTATCTTCAGGCGGC \\
\hline $\mathrm{K} 32$ & 143 & ATGAGGGTAGGGAAAGGGGTATCTCGGCGG \\
\hline K33 & 136 & GGGCACGGTGGAGTGGGGGTCCTTCCTGGG \\
\hline K34 & 132 & GAGGGTCAGGGATTTGGGGTATCTGGGTGG \\
\hline K35 & 127 & TTGGAGTGGTGGGTGGGGATCGTGAGGCGG \\
\hline K36 & 124 & ACACGGTGGTGTGGGGGTTTCCAGGGCGGG \\
\hline $\mathrm{K} 37$ & 121 & CTGTCGGTGCCCACGGTGGAGAGGGGGTGG \\
\hline K38 & 117 & TGAGGGAGGGACAGGGGTATCTTGGTGCGG \\
\hline K39 & 116 & CCCTGTCACGGTGGTGTGGGGGTATAGGGC \\
\hline K40 & 114 & AGAGGGAGGGCAAGGGTTCTACCAGGTCG \\
\hline K41 & 113 & CCAGCACGCGGTGGAGAGGGGGATGTTGGC \\
\hline K42 & 111 & TGGCCGATCACGGAGGAGAGGGGGTATCGG \\
\hline K43 & 106 & GAGGGTTAAGGGACGGGGTATCTGAGGCGG \\
\hline K44 & 97 & GTGGGATAGGGTTTACGGGGTATCGGTGGG \\
\hline K45 & 94 & CACGGAGGAGAGGGGGTTCCATCGTTGTCG \\
\hline K46 & 92 & TTGAGGTGGGATAGGGTAGGGGTCGTGTGC \\
\hline K47 & 92 & GTGGGAGGGTGGGCTCTGCCAGAACCGGC \\
\hline K48 & 92 & AGTGGGTTGGGTAATGGGGTATCTACGGGG \\
\hline K49 & 88 & CACGGTGGAGAGGGGGATCCTACTACTCGG \\
\hline K50 & 88 & ACAGTGGGAGGGAGGGTTATCACCGGGCCG \\
\hline
\end{tabular}



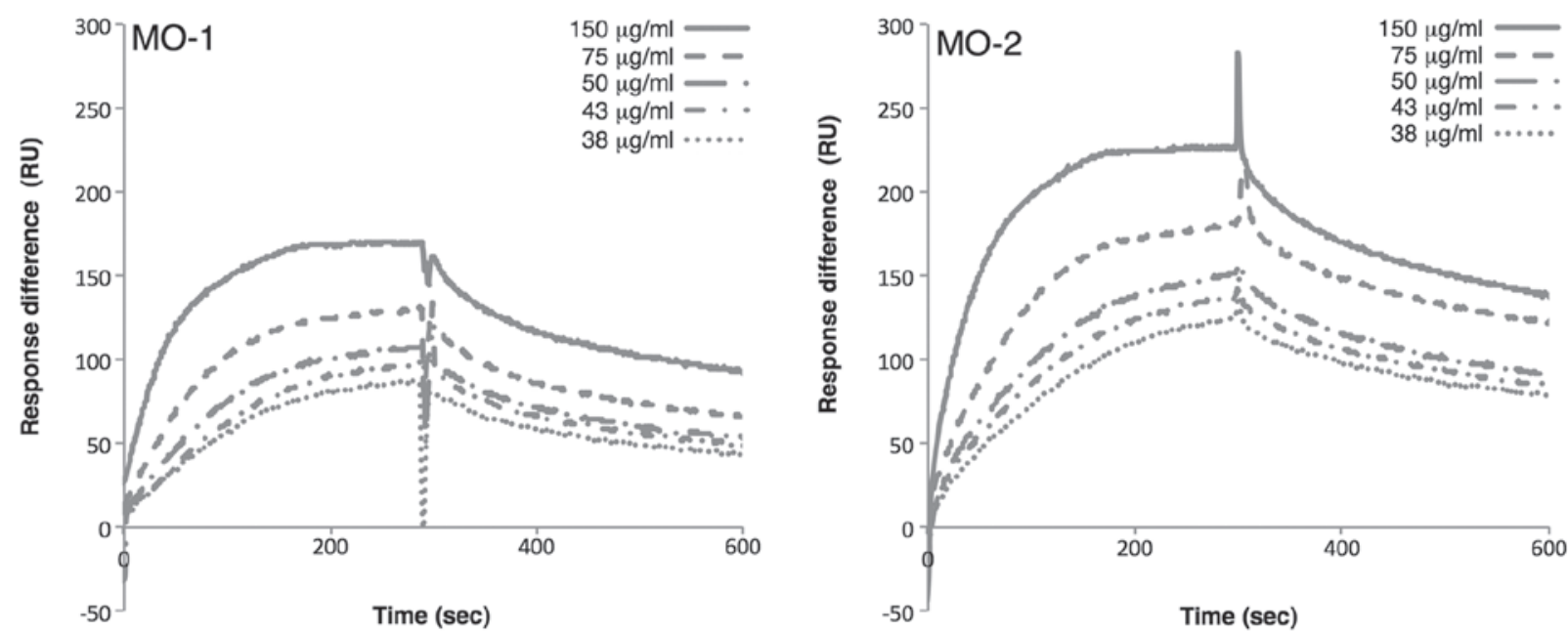

Figure 3. Analysis by SPR spectroscopy of binding of aptamers to extracellular vesicles. The binding affinity of aptamers was examined by SPR spectroscopy with the Biacore $\mathrm{X}$ system. Extracellular vesicles at various concentrations $(150,75,50,43$ and $38 \mu \mathrm{g} / \mathrm{ml})$ were passed over aptamers, which had been immobilized on streptavidin-coated sensor chips. The sensorgrams demonstrate the significant binding of the aptamers to extracellular vesicles. RU, resonance units; SPR, surface plasmon resonance.

analysis indicated that the binding affinities of both aptamers increased in the presence of potassium ions (Fig. 5), reflecting the observation that the G-quadruplex structure is stabilized by potassium ions. The strong affinities of the aptamers for extracellular vesicles may be sustained by the tight structure of the G-quadruplex.

Properties of extracellular vesicles recognized by aptamer $M O-1$. Extracellular vesicles, including exosomes and microvesicles, exist as heterogeneous populations of vesicles of different sizes, densities and constituent molecules because of difference in origin and mode of generation (37). Therefore, whether the MO-1 aptamer recognized all fractions of extracellular vesicles or only some members of populations of vesicles was investigated. The authors fractioned extracellular vesicles in terms of size on a sepharose $2 \mathrm{~B}$ column and monitored the distribution of amounts of total protein (upper graph in Fig. 6A) and CD63 protein, a marker of exosomes (middle panel in Fig. 6A). The CD63-specific antibody bound strongly around fraction 4 . By contrast, aptamer MO-1 exhibited strong affinity for fractions 3 through 6 (lower panel in Fig. 6A). Thus, the aptamer appeared to recognize the population of vesicles that included a majority of exosomes.

Also examined was the binding of the MO-1 aptamer to denatured extracellular vesicles (Fig. 6B). The binding affinity of the aptamer (MO-1) for heat-denatured and for SDS-denatured extracellular vesicles was very much reduced. Thus, it seems likely that the aptamer may recognize a specific protein structure on the extracellular vesicles.

\section{Discussion}

This study is the first, to the best of the authors' knowledge, to identify RNA aptamers (33-nucleotide MO-1 and 38-nucleotide MO-2) that bind to extracellular vesicles. The sequences were identified with a next-generation sequencer and each consisted of a non-conserved stem with a conserved loop
(Fig. 2D). SPR analysis revealed that the aptamers MO-1 and MO-2 had similar strong affinity for extracellular vesicles (Fig. 3). Structural analysis by CD spectroscopy demonstrated that both aptamers formed a G-quadruplex in the loop region (Fig. 4).

The two aptamers MO-1 and MO-2, with consensus sequence-1, were obtained from two independent libraries, in which the lengths of the randomized regions and the sequences of fixed primer regions were completely different (see Materials and methods). This result may reflect the fact that the aptamers were effectively and efficiently selected from a huge variety of randomized sequences. Alternatively, it is possible that the presence of consensus sequence- 1 in both aptamers was due to its strong affinity and shorter length.

All the candidate aptamers with consensus sequence- 1 and consensus sequence-2, respectively, had non-conserved stem regions. In other words, the regions surrounding the consensus sequences were different but were able to form stable double-strand structures. This method allowed the authors to concentrate related sequences from a massive diversity of libraries sequences effectively, comprehensively and individually.

Spectroscopic analysis suggested that the MO-1 and MO-2 aptamers formed a G-quadruplex structure in their loop regions, and the stable conformation of the G-quadruplex in the presence of potassium ions may contribute to the binding affinity of the aptamers for extracellular vesicles (Fig. 5). The stem-G-quadruplex structure of these aptamers may be one of essential structures for recognition of target molecules as a binding scaffold with strong affinity and compact size.

To confirm the interaction between MO-1 and extracellular vesicles, the authors designed 'pull-down' experiments using the 5'-biotinylated MO-1 aptamer. In spite of several attempts, these experiments failed, presumably because of difficulties in capturing vesicles of high molecular weight (data not shown). The binding of MO-1 to size-fractionated extracellular vesicles suggested that a majority of its targets may be exosomes but the targets may also be other entities of 

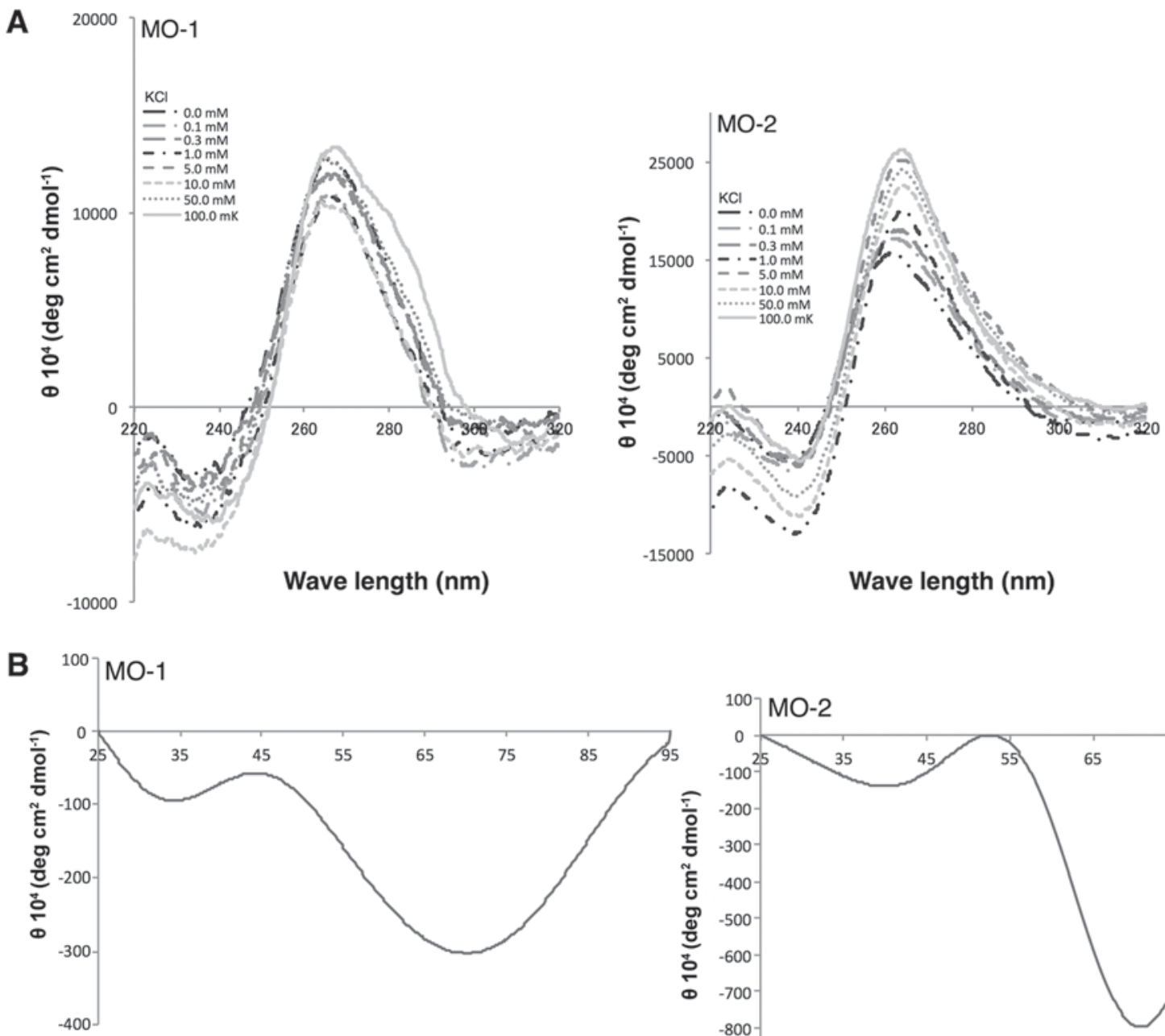

Temperature $\left({ }^{\circ} \mathrm{C}\right)$
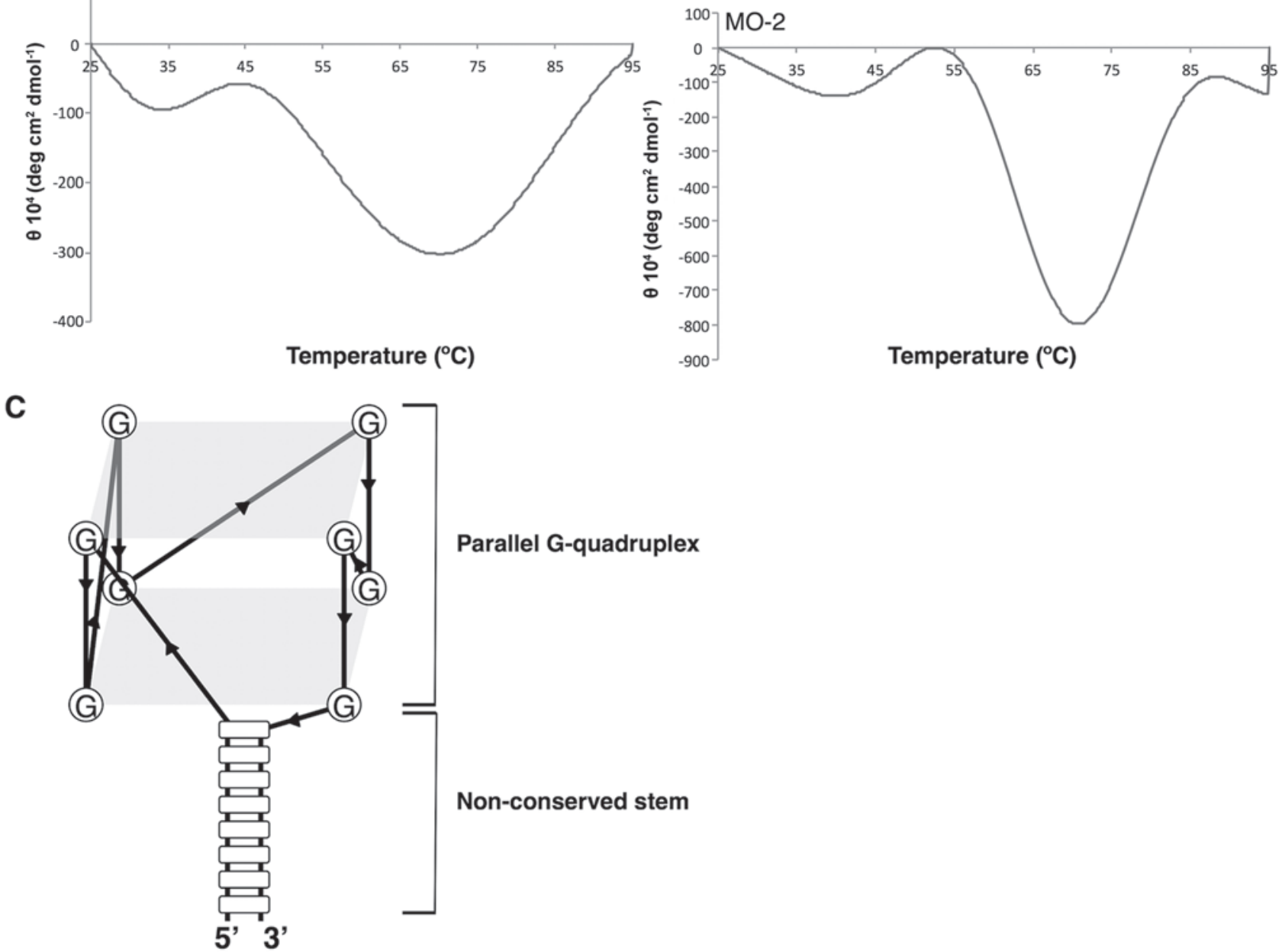

Parallel G-quadruplex

Non-conserved stem

Figure 4. Analysis of quadruplex structures. (A) CD spectra of aptamers at various concentrations of $\mathrm{KCl}(0,0.1,0.3,1,5,10,50$ and $100 \mathrm{mM})$. The aptamers yielded a negative peak at $240 \mathrm{~nm}$ and a positive peak at $260 \mathrm{~nm}$, an indication of the formation of a parallel quadruplex structure. (B) CD melting-temperature curves of aptamers at $270 \mathrm{~nm}$ in $100 \mathrm{mM} \mathrm{KCl}$. (C) Putative G-quadruplex structure of the aptamers. The loop region of the hairpin structure folds into the G-quadruplex structure. $\mathrm{CD}$, circular dichroism; $\mathrm{MO}$, minimized oligonucleotides; $\mathrm{KCl}$, potassium chloride.

similar molecular weight. The SPR analyses demonstrated that extracellular vesicles derived from HeLa S3 cells also bound to the MO-1 aptamer, suggesting that the same molecule(s) derived from both 293T and HeLa S3 cells may be its target (Fig. 3). Moreover, binding affinity was severely depressed under denaturing conditions (Fig. 6B). Thus, it is very likely 

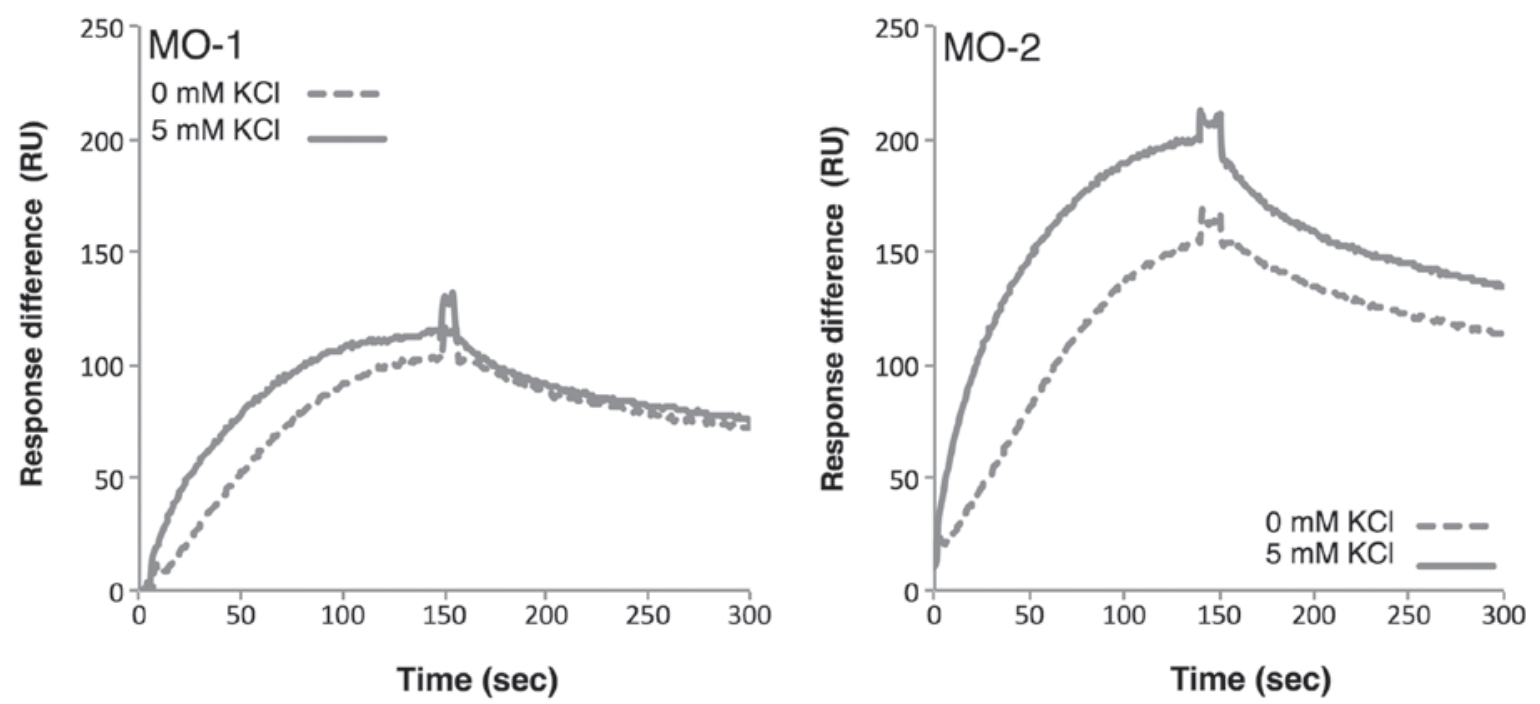

Figure 5. Dependence of changes in the SPR spectra of aptamers on the concentration of potassium ions. The affinities of aptamers for extracellular vesicles $(75 \mu \mathrm{g} / \mathrm{ml})$ were analyzed by SPR spectroscopy in the presence or absence of potassium ions $(5 \mathrm{mM})$. SPR, surface plasmon resonance; MO, minimized oligonucleotides; $\mathrm{KCl}$, potassium chloride; $\mathrm{RU}$, resonance units.

\section{A}

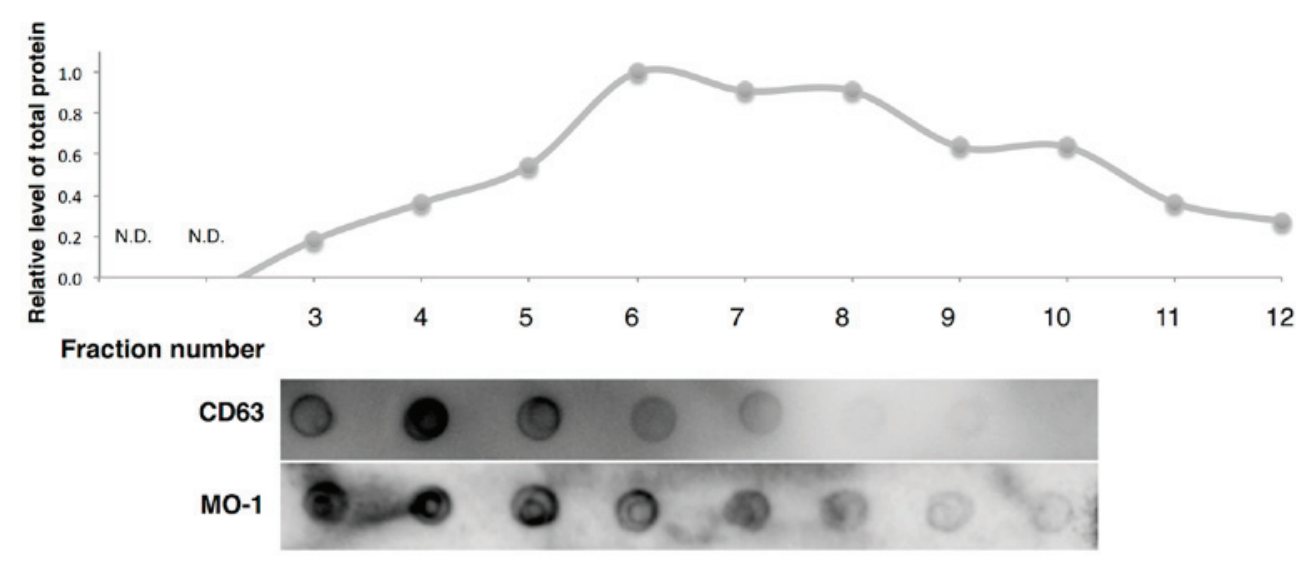

B

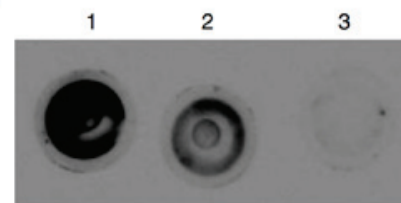

1: Extracellular vesicles

2: Boiled extracellular vesicles

3: Boiled extracellular vesicles

in buffer that included $10 \%$ SDS

Figure 6. Properties of extracellular vesicles that were recognized by aptamer MO-1. (A) Extracellular vesicles were fractionated by on a column of sepharose 2B. The distribution of proteins (by mass) in the fractionated extracellular vesicles (fraction numbers 1-12) is presented in the graph. Results of blotting analysis, indicating the affinities of CD63 and the aptamer for the fractions of extracellular vesicles, are presented in the middle panel and the lower panel, respectively. (B) The affinity of the MO-1 aptamer for denatured extracellular vesicles was evaluated by a dot-blotting assay. MO, minimized oligonucleotides.

that the targets of the MO-1 and MO-2 aptamers are proteins on the surface of extracellular vesicles, for example, CD9, CD13, CD26, CD63, CD81, heat shock protein 70, integrins, intercellular adhesion molecule-1, major histocompatibility complex and tumor necrosis factor (38-41).

Previously, a patent was filed for a sequence very similar to that of MO-1 (42). The sequence was identified as an aptamer that bound to platelet-derived growth factor (PDGF). In the patent application, the claim was made that the sequence was able to bind to PDGF receptors, to vascular endothelial growth factor and its receptors. Therefore, the target of MO-1 may be such a ligand or receptor on extracellular vesicles.

For development of diagnostic technologies that exploit extracellular vesicles, aptamers have several advantages over antibodies. The most significant advantage is that extracellular vesicles can be purified intact, under mild conditions, from body fluids (11). Captured vesicles can be eluted from an aptamer-conjugated support by chelating agents, such as EDTA, which can unfold aptamers by sequestering magnesium ions. In addition, aptamers can target small molecules, 
lipids and sugars (18). Aptamers can be easily improved by inclusion of modified nucleic acids or artificial nucleic acids that enhance stability and/or the ability to bind to nucleic acids. Furthermore, a method using counter-SELEX (43) may allow isolation of aptamers against disease-specific extracellular vesicles via 'subtractive' use of disease-derived and normal vesicles. In such counter-SELEX, the aptamers identified in the present study, which recognize whole extracellular vesicles, may be useful in the future as blocking reagents.

It is hoped that the current work will lead to the development of extracellular vesicle-based diagnostic systems in which aptamers are used to target disease-specific molecules on extracellular vesicles.

\section{Acknowledgements}

The present study was supported by a Grant-in-Aid for Challenging Exploratory Research from the Japan Society for the Promotion of Science (grant no. 26640097). The authors would like to thank Dr M. Ikemoto for his helpful comments and Dr T. Yamasaki for her skilled technical assistance.

\section{References}

1. Simpson RJ, Jensen SS and Lim JW: Proteomic profiling of exosomes: Current perspectives. Proteomics 8: 4083-4099, 2008.

2. Lee Y, El Andaloussi S and Wood MJ: Exosomes and microvesicles: Extracellular vesicles for genetic information transfer and gene therapy. Hum Mol Genet 21: R125-R134, 2012.

3. Valadi H, Ekström K, Bossios A, Sjostrand M, Lee JJ and Lötvall JO: Exosome-mediated transfer of mRNAs and microRNAs is a novel mechanism of genetic exchange between cells. Nat Cell Biol 9: 654-659, 2007.

4. Skog J, Wurdinger T, van Rijn S, Meijer DH, Gainche L, Sena-Esteves M, Curry WT Jr, Carter BS, Krichevsky AM and Breakefield XO: Glioblastoma microvesicles transport RNA and proteins that promote tumour growth and provide diagnostic biomarkers. Nat Cell Biol 10: 1470-1476, 2008

5. Simons M and Raposo G: Exosomes-vesicular carriers for intercellular communication. Curr Opin Cell Biol 21: 575-581, 2009.

6. Taylor DD and Gercel-Taylor C: MicroRNA signatures of tumor-derived exosomes as diagnostic biomarkers of ovarian cancer. Gynecol Oncol 110: 13-21, 2008.

7. Zhou H, Pisitkun T, Aponte A, Yuen PS, Hoffert JD, Yasuda H, Hu X, Chawla L, Shen RF, Knepper MA and Star RA: Exosomal Fetuin-A identified by proteomics: A novel urinary biomarker for detecting acute kidney injury. Kidney Int 70: 1847-1857, 2006.

8. Yamashita T, Kamada H, Kanasaki S, Maeda Y, Nagano K, Abe Y, Inoue M, Yoshioka Y, Tsutsumi Y, Katayama S, et al: Epidermal growth factor receptor localized to exosome membranes as a possible biomarker for lung cancer diagnosis. Pharmazie 68: 969-973, 2013.

9. Zoller M: Pancreatic cancer diagnosis by free and exosomal miRNA. World J Gastrointest Pathophysiol 4: 74-90, 2013.

10. Melo SA, Luecke LB, Kahlert C, Fernandez AF, Gammon ST, Kaye J, LeBleu VS, Mittendorf EA, Weitz J, Rahbari N, et al: Glypican-1 identifies cancer exosomes and detects early pancreatic cancer. Nature 523: 177-182, 2015.

11. Thery C, Amigorena S, Raposo G and Clayton A: Isolation and characterization of exosomes from cell culture supernatants and biological fluids. Curr Protoc Cell Biol: Chapter 3:Unit 3.22, 2006.

12. Rekker K, Saare M, Roost AM, Kubo AL, Zarovni N, Chiesi A, Salumets A and Peters M: Comparison of serum exosome isolation methods for microRNA profiling. Clin Biochem 47: 135-138, 2014.

13. van der Pol E, Hoekstra AG, Sturk A, Otto C, van Leeuwen TG and Nieuwland R: Optical and non-optical methods for detection and characterization of microparticles and exosomes. J Thromb Haemost 8: 2596-2607, 2010.

14. Grant R, Ansa-Addo E, Stratton D, Antwi-Baffour S, Jorfi S, Kholia S, Krige L, Lange S and Inal J: A filtration-based protocol to isolate human plasma membrane-derived vesicles and exosomes from blood plasma. J Immunol Methods 371: 143-151, 2011.
15. Tauro BJ, Greening DW, Mathias RA, Ji H, Mathivanan S, Scott AM and Simpson RJ: Comparison of ultracentrifugation, density gradient separation, and immunoaffinity capture methods for isolating human colon cancer cell line LIM1863-derived exosomes. Methods 56: 293-304, 2012.

16. Ellington $\mathrm{AD}$ and Szostak JW: In vitro selection of RNA molecules that bind specific ligands. Nature 346: 818-822, 1990.

17. Tuerk C and Gold L: Systematic evolution of ligands by exponential enrichment: RNA ligands to bacteriophage T4 DNA polymerase. Science 249: 505-510, 1990.

18. Stoltenburg R, Reinemann C and Strehlitz B: SELEX-a (r)evolutionary method to generate high-affinity nucleic acid ligands. Biomol Eng 24: 381-403, 2007.

19. Ohuchi SP, Ohtsu T and Nakamura Y: Selection of RNA aptamers against recombinant transforming growth factor-beta type III receptor displayed on cell surface. Biochimie 88: 897-904, 2006.

20. Daniels DA, Chen H, Hicke BJ, Swiderek KM and Gold L: A tenascin-C aptamer identified by tumor cell SELEX: Systematic evolution of ligands by exponential enrichment. Proc Natl Acad Sci USA 100: 15416-15421, 2003.

21. Wilner SE, Wengerter B,Maier K, de Lourdes Borba Magalhães M, Del Amo DS, Pai S, Opazo F, Rizzoli SO, Yan A and Levy M: An RNA alternative to human transferrin: A new tool for targeting human cells. Mol Ther Nucleic Acids 1: e21, 2012.

22. Li N, Nguyen HH, Byrom M and Ellington AD: Inhibition of cell proliferation by an anti-EGFR aptamer. PLoS One 6: e20299, 2011.

23. Shigdar S, Lin J, Yu Y, Pastuovic M, Wei M and Duan W: RNA aptamer against a cancer stem cell marker epithelial cell adhesion molecule. Cancer Sci 102: 991-998, 2011.

24. Sekiya S, Noda K, Nishikawa F, Yokoyama T, Kumar PK and Nishikawa S: Characterization and application of a novel RNA aptamer against the mouse prion protein. J Biochem 139: 383-390, 2006

25. Murakami K, Nishikawa F, Noda K, Yokoyama $T$ and Nishikawa S: Anti-bovine prion protein RNA aptamer containing tandem GGA repeat interacts both with recombinant bovine prion protein and its beta isoform with high affinity. Prion 2: 73-80, 2008.

26. Nishikawa F, Murakami K, Matsugami A, Katahira M and Nishikawa S: Structural studies of an RNA aptamer containing GGA repeats under ionic conditions using microchip electrophoresis, circular dichroism and 1D-NMR. Oligonucleotides 19: 179-190, 2009.

27. Hamada M, Sato K, Kiryu H, Mituyama T and Asai K: Predictions of RNA secondary structure by combining homologous sequence information. Bioinformatics 25: i330-i338, 2009.

28. Bailey TL, Boden M, Buske FA, Frith M, Grant CE, Clementi L, Ren J, Li WW and Noble WS: MEME SUITE: Tools for motif discovery and searching. Nucleic Acids Res 37 (Web Server issue): W202-W208, 2009.

29. Kikin O, D'Antonio L and Bagga PS: QGRS Mapper: A web-based server for predicting G-quadruplexes in nucleotide sequences. Nucleic Acids Res 34: W676-W682, 2006.

30. Matsugami A, Ouhashi K, Kanagawa M, Liu H, Kanagawa S, Uesugi $S$ and Katahira M: An intramolecular quadruplex of (GGA)(4) triplet repeat DNA with a G:G:G:G tetrad and a $\mathrm{G}(: \mathrm{A}): \mathrm{G}(: \mathrm{A}): \mathrm{G}(: \mathrm{A}): \mathrm{G}$ heptad and its dimeric interaction. J Mol Biol 313: 255-269, 2001

31. Catasti P, Chen X, Moyzis RK, Bradbury EM and Gupta G: Structure-function correlations of the insulin-linked polymorphic region. J Mol Biol 264: 534-545, 1996.

32. Xia T, SantaLucia J Jr, Burkard ME, Kierzek R, Schroeder SJ, Jiao X, Cox C and Turner DH: Thermodynamic parameters for an expanded nearest-neighbor model for formation of RNA duplexes with Watson-Crick base pairs. Biochemistry 37: 14719-14735, 1998.

33. Mashima T, Matsugami A, Nishikawa F, Nishikawa S and Katahira M: Unique quadruplex structure and interaction of an RNA aptamer against bovine prion protein. Nucleic Acids Res 37: 6249-6258, 2009.

34. Mashima T, Nishikawa F, Kamatari YO, Fujiwara H, Saimura M, Nagata T, Kodaki T, Nishikawa S, Kuwata K and Katahira M: Anti-prion activity of an RNA aptamer and its structural basis. Nucleic Acids Res 41: 1355-1362, 2013.

35. Matsugami A, Mashima T, Nishikawa F, Murakami K, Nishikawa S, Noda K, Yokoyama T and Katahira M: Structural analysis of $\mathrm{r}(\mathrm{GGA}) 4$ found in RNA aptamer for bovine prion protein. Nucleic Acids Symp Ser (Oxf): 79-180, 2008. 
36. Zhang AY, Bugaut A and Balasubramanian S: A sequence-independent analysis of the loop length dependence of intramolecular RNA G-quadruplex stability and topology. Biochemistry 50: 7251-7258, 2011

37. Kowal J, Arras G, Colombo M, Jouve M, Morath JP, Primdal-Bengtson B, Dingli F, Loew D, Tkach M and Théry C: Proteomic comparison defines novel markers to characterize heterogeneous populations of extracellular vesicle subtypes. Proc Natl Acad Sci USA 113: E968-E977, 2016.

38. Christianson HC, Svensson KJ, van Kuppevelt TH, Li JP and Belting M: Cancer cell exosomes depend on cell-surface heparan sulfate proteoglycans for their internalization and functional activity. Proc Natl Acad Sci USA 110: 17380-17385, 2013

39. Thery C, Ostrowski M and Segura E: Membrane vesicles as conveyors of immune responses. Nat Rev Immunol 9: 581-593, 2009.
40. Hoshino A, Costa-Silva B, Shen TL, Rodrigues G, Hashimoto A, Tesic Mark M, Molina H, Kohsaka S, Di Giannatale A, Ceder S, et al: Tumour exosome integrins determine organotropic metastasis. Nature 527: 329-335, 2015.

41. Clayton A, Court J, Navabi H, Adams M, Mason MD, Hobot JA, Newman GR and Jasani B: Analysis of antigen presenting cell derived exosomes, based on immuno-magnetic isolation and flow cytometry. J Immunol Methods 247: 163-174, 2001.

42. Epstein D, Grate D, Stanton M, et al: Stabilized aptamers to platelet derived growth factor and their use as oncology therapeutics. US Patent No US20040253679 A1 Filed April 21, 2004; issued December 16, 2004.

43. Jenison RD, Gill SC, Pardi A and Polisky B: High-resolution molecular discrimination by RNA. Science 263: 1425-1429, 1994. 